\title{
¿Cómo hacer del aprendizaje de la biología un asunto relevante para los estudiantes?
}

\section{How to make Biology learning an outstanding affair for students?}

\section{Por: Correa Alejandra ${ }^{1}$}

\section{Resumen}

En este ensayo se proponen algunas ideas generales para mejorar la enseñanza de la biología. Estas incluyen el uso de situaciones de la vida real que les permitan a los estudiantes conectar su experiencia cotidiana con el conocimiento biológico, y el desarrollo de una discusión en clase sobre algunos aspectos de la historia de la biología, en los cuales se muestre una visión más realista del proceso de construcción del conocimiento biológico.

Palabras clave: Enseñanza de la biología, historia de la biología, contexto social, contenido interdisciplinar.

\begin{abstract}
In this essay some general ideas to improve the teaching of biology are proposed. These include the use of real live situations which allow the students to connect their everyday experience with the biological knowledge, and the development of a class discussion about some aspects from the biology history, which show a more realistic picture of the building process of biological knowledge.
\end{abstract}

Keywords: Biology teaching, biology history, social context, interdisciplinary content.

La biología es un componente fundamental de la vida cotidiana, ya que hace parte de los titulares de prensa (nuevas terapias génicas, epidemias, problemas ecológicos, etc.), así como de nuestro entorno más inmediato (alimentos, mascotas, plantas, salud, entre otros), por sólo mencionar algunos ejemplos. Lo anterior muestra el valor estratégico de la enseñanza de la biología para el desarrollo de las naciones, lo cual aparece reflejado en la siguiente afirmación aplicada a las ciencias en general: "En efecto, la tradicional importancia concedida a las inversiones en educación científica y tecnológica, para hacer posible el desarrollo futuro de un país, ha dejado paso al convencimiento de que la alfabetización científica de todos los ciudadanos y ciudadanas ha pasado a constituir una exigencia urgente, un requisito también para el desarrollo inmediato" (Gil et al., 2000, p. 13). De ahí, la importancia de enseñar a los estudiantes a hacer conexiones entre lo que ellos aprenden en el salón de clase y lo que encuentran en la vida diaria. Para cumplir con este objetivo “... los problemas que se deberían trabajar en la escuela son los problemas relevantes para los ciudadanos, no los problemas científicos, de forma que la ciencia queda al servicio de lo social" (García, 1998, p. 68).

${ }^{1}$ Estudiante de la Especialización en Enseñanza de la Biología, Universidad Pedagógica Nacional. Bogotá, Colombia. 2009. acorream@gmail.com. 
Las implicaciones de hacer ciencia en el vacío son muy perniciosas, ya que no existe la denominada "ciencia neutra"; de ahí que todo investigador deba responder ante la sociedad, mediante una comunicación efectiva con las legislaciones locales y nacionales. "Por otra parte, casi nadie duda ya de que la ciencia y la tecnología forman parte de un complejo entramado social, político y económico que no suele explicitarse en su enseñanza, con lo cual se favorece, aun más, la imagen de desarraigo del trabajo científico y se potencia la imagen de la ciencia como un producto de la genialidad individual o de unos pocos" (Arnay, 1997, p. 43).

Por ello no es de extrañar que, con frecuencia, el ciudadano común se siente incapaz de controlar ciertos productos tecnológicos o de afrontar simples razonamientos relacionados con la ciencia. La educación debería disminuir esa inseguridad de tal forma que se pudiera disfrutar de los crecientes beneficios de la era de la ciencia y la tecnología, garantizando la protección de la salud y el medio, y contribuyendo a la toma de decisiones sobre el desarrollo científico y tecnológico. Por tanto, la alfabetización científica será necesaria para contribuir a formar ciudadanos que sepan desenvolverse en un mundo como el actual y que conozcan el importante papel que la ciencia desempeña en sus vidas y en la sociedad (Aikenhead, 1985; Bingle y Gaskell, 1994; Gil et al., 1991; Solbes y Vilches, 1997). Desde el punto de vista didáctico, esto significa que "la solución al fracaso de la enseñanza no pasa por divorciar aún más la escuela de la vida cotidiana sino por dejar de considerar a las disciplinas científicas como el único marco de referencia en la escuela" (García, 1998, p. 56).

Precisamente, este fracaso en la enseñanza se debe a que los contenidos de los programas de las diferentes instituciones educativas tienen “... un valor y sentido igualmente alejado de lo cotidiano y de lo científico. De lo cotidiano por el hecho de que no está previsto que su obtención sirva para la reflexión y acción en la vida cotidiana, puesto que, para ello las personas elaboran modelos implícitos que sirven para interpretar los fenómenos que acontecen en las dimensiones intermedias de la realidad (mesomundo), mientras el conocimiento académico trata de transmitir, principalmente, los modelos y teorías científicas sobre dimensiones del micro y macromundo" (Arnay, 1997, p. 35).

En el presente ensayo, se plantea como posible solución a este problema la enseñanza de una biología dedicada al entorno social, lo cual permitiría a los estudiantes hacer, por sí mismos, las conexiones entre su saber cotidiano y el conocimiento biológico. De este modo, al integrar los temas sociales en el currículo de biología, se generará una responsabilidad social por los asuntos biológicos que hará evidente la necesidad de una alfabetización en biología para todos. En otras palabras lo que se busca es facilitar "la consecución de algunos grandes fines educativos: dotar a las personas y a los grupos sociales de una visión de conjunto del mundo que les permita comprender y actuar en la realidad en que viven; de unos recursos que les capaciten para el ejercicio de la autonomía, la cooperación la creativa y la libertad; de una formación que facilite la investigación de su entorno y la reflexión sobre su propia práctica, no sólo en el ámbito escolar, sino también en los demás ámbitos de su vida cotidiana" (García, 1998, p. 16).

Otra ventaja de esta integración es que se reduce al mínimo la probabilidad de que se produzca el fenómeno denominado por Chevallard como desgaste del saber enseñado. "Se trata de un desgaste que podemos considerar "biológico" y que lo aleja demasiado visiblemente del saber sabio... Con el tiempo, el saber tratado por el sistema de enseñanza envejece; un buen día se percibe que se ha vuelto 
viejo en relación a la sociedad (en relación con el saber sabio y el saber banalizado). Por un lado envejecimiento biológico- se lo declara en desacuerdo con el desarrollo del saber correspondiente en sus formas libres (no escolarizadas). Desacuerdo que puede comprender contenidos diversos: puede ocurrir que como corolario del progreso de la investigación se revelen como falsos los resultados hasta entonces enseñados... o puede ocurrir incluso que cierta cuestión que ocupaba un lugar importante en los programas, bruscamente se considere carente de interés a la luz de nuevos desarrollos o cambios de las problemáticas del campo científico considerado... Por otro lado -envejecimiento "moral"- el saber enseñado se encontrará en desacuerdo con la sociedad en un sentido amplio, aunque, llegado el caso, si se lo juzgara estrictamente según los criterios de la disciplina correspondiente no habría nada que reprocharle. En resumen, una cuestión de época o de estado de ánimo" (Chevallard, 1991, pp. 30-31).

En el caso de la enseñanza de la biología, se tiene la gran ventaja de que la mayoría de los progresos de esta disciplina afectan directamente el entorno social, lo cual hace que esta integración genere un saber enseñado relevante y en mayor concordancia con los problemas de la sociedad moderna, tal como se observa en el caso de la relación entre ciencia, tecnología sociedad y ambiente (CTSA). Por lo tanto, la función del docente sería la de involucrar a lo educandos, generando una actitud de apropiación y de búsqueda de soluciones a los conflictos de la interacción CTSA. "Por eso, en la medida en que la gestión de los problemas socioambientales (salud, planificación del territorio, contaminación, marginación de las minorías, mejora de la calidad de vida, control demográfico, etc.) no es únicamente responsabilidad del experto, sino de todos los ciudadanos, resulta imprescindible ampliar la noción de "lo cotidiano" a toda esta problemática" (García, 1998, p. 42).

Sin embargo, para muchos docentes, el incremento de los contenidos disciplinares hace que sea todo un desafío encontrar un espacio para integrar los temas sociales en las clases de biología sin omitir, en el proceso, algunos de los contenidos biológicos. Una buena estrategia consiste en presentar problemas del contexto social en aquellos tópicos que se revisen repetidamente en los diferentes cursos de biología, lo cual reduciría al mínimo cualquier sacrificio en tiempo o contenidos, y permitiría a los alumnos reflexionar sobre su entorno social más de una vez. De esta forma, al extender el marco conceptual biológico a los temas de relevancia social, se puede mejorar la retención de lo aprendido por los educandos, ya que cada concepto ha tenido múltiples puntos de entrada y, por ende, múltiples puntos de interés que sirven de vías para la recuperación de la información. Con esto, "la ciencia, tanto desde el análisis de la naturaleza de los contenidos científicos en sí mismos (perspectiva de la lógica de las disciplinas en cuestión), como desde la perspectiva de la historia de dichas disciplinas, constituye una fuente de aportaciones de primer orden al análisis de los problemas socioambientales" (García, 1998, p. 19).

Lo anterior, esta en completa concordancia con la tendencia en la didáctica de las ciencias de promover "una enseñanza de las ciencias para todos los ciudadanos como medio para democratizar el uso social y político de la ciencia. Las personas, según este nuevo punto de vista, deben comprender para poder decidir, deben desmitificar, para poder participar y deben incorporar en su formación básica ciertos aspectos de la cultura científica. Se comienza, por tanto, a cuestionar si el objetivo de la educación científica en las etapas obligatorias es realmente «enseñar las ciencias en sentido estricto» o, más bien, contribuir desde las ciencias a la formación general de todos los ciudadanos" (Porlán, 1998, p. 178). 
La mejor forma de implementar lo expuesto anteriormente, sería usando problemas reales que conecten varios conceptos de la biología; esto promovería en los estudiantes una visión más crítica y compleja de la biología que los aleje del imaginario de esta disciplina como colección de datos desconectados y apartados de la realidad, la cual se deriva de los enfoques transmitivos tan criticados por la didáctica de las ciencias. Este cuestionamiento a la idea promovida por la enseñanza tradicional de que los alumnos son simples receptores pasivos de la información, es el resultado de los avances de los estudios sobre las concepciones de los estudiantes, que señalan su naturaleza espontánea y, con frecuencia, distinta a la ofrecida por la ciencia. Por lo tanto, "la existencia de estas concepciones... refuerza la idea de que es necesario teorizar sobre la naturaleza epistemológica del conocimiento escolar, pues ya no es posible considerar la ciencia como única fuente de contenidos, dado que los alumnos contienen también una visión del mundo que el currículo debe considerar" (Porlán, 1998, p. 181).

Este giro en el énfasis en los contenidos, desde lo meramente disciplinar a la integración de varios conocimientos, se basa en numerosas investigaciones de la ciencia cognitiva que demuestran cómo el conocimiento previo de los educandos, puede influenciar la forma en que se organiza y se conecta la información nueva, mediante la construcción de esquemas profundamente enraizados en las experiencias personales y culturales (Vygotsky, 1978; Ausubel et al., 1978; Lattuca et al., 2004). "Evidentemente, el pensamiento de "sentido común" es el punto de partida, pero no para sustituirlo por el científico o para ignorarlo, sino para enriquecerlo, para hacerlo complejo" (García, 1998, p. 21).

En varios trabajos se recomienda este tipo de enfoque pedagógico porque se ha visto que prepara mejor a los alumnos para enfrentarse a la naturaleza interdisciplinaria de los problemas del siglo XXI (National Research Council [NRC], 2005). De ahí, la importancia de “... considerar la diversidad de formas de conocimiento implicadas en la determinación del conocimiento escolar. En ese sentido, no se puede ignorar que hay formas de conocimiento metadisciplinares, de carácter más general y descontextualizado, que deberían ser un referente fundamental para la determinación del conocimiento escolar, sobre todo si queremos propiciar cambios en la perspectiva epistemológica de los sujetos, en su manera de ver el mundo" (García, 1998, p. 61).

Para el caso particular de la enseñanza de la biología, estos conceptos metadisciplinares servirían de criterio para escoger los problemas del ámbito social que favorezcan de forma más efectiva el desarrollo de un pensamiento holístico, basado en una visión sistémica del mundo. A esto se suma el hecho de que, "... el conocimiento metadisciplinar debe tener un papel relevante en la selección y organización del conocimiento escolar, de manera que las aportaciones del conocimiento científico y cotidiano, relativas a cierto problema o tópico, se interpreten en función de dicho marco de referencia más general. Así el conocimiento metadisciplinar es útil para dar cierto sentido al tratamiento de los objetos de estudio, y para establecer el grado de complejidad en el que deben ser formulados los contenidos para que puedan ser aprendidos" (García, 1998, p. 72).

Con base en lo anterior, se plantea que, "... la escuela debe convertirse en un lugar de reflexión sobre las relaciones entre los humanos, y entre éstos y el medio, y en un motor de cambio social, teniendo como objeto educativo básico lo que podríamos denominar el enriquecimiento del conocimiento cotidiano. La evolución de dicho conocimiento se dirigiría así hacia la construcción, por parte de los sujetos, de un determinado modelo de desarrollo humano (individual y colectivo) alternativo al actualmente 
predominante" (García, 1998, p. 16). Para cumplir con este ideal se debe “... ir más allá de la distinción, en el conocimiento escolar, entre problemas científicos y problemas cotidianos, mediante la propuesta de problemas que son cotidianos en la medida en que afectan a nuestras vidas, pero que por su complejidad requieren, en su tratamiento, de la participación de otras formas de conocimiento" (García, 1998, p. 17).

A continuación, se mencionan algunas estrategias generales, de carácter interdisciplinar, que favorecen una reflexión más compleja de la realidad por parte de los estudiantes, mediante la aplicación de conceptos de la disciplina de la biología y de su historia y epistemología, a la experiencia cotidiana.

Las biografías de los principales protagonistas de la biología les recuerdan a los estudiantes que las investigaciones biológicas son una empresa humana que, como cualquier otra, no está aislada de la política, las normas sociales o los paradigmas de la época (factores externos). Así mismo, para mostrarles que estas mismas investigaciones pueden ser influenciadas por los mismos miembros de la comunidad científica (factores internos), los docentes pueden acudir a la historia de la biología para describir el contexto disciplinar en el que se desarrollaron principios clave, métodos y conceptos. Al trazar el camino de la ciencia a lo largo de la historia, el alumno verá que el conocimiento biológico es el resultado de la actividad humana en la que cada investigador construye a partir del trabajo de otros, por medio de la comunicación, la competencia y la colaboración. Además, estas experiencias tienen un gran valor didáctico ya que “... historias como éstas pueden tener cierto carácter de prácticas. Observamos cómo los conocimientos pueden reelaborarse en forma de relatos, no simplemente para añadir su «vivacidad» o su «interés» ni simplemente para «aplicarlo» a algún contexto real, sino fundamentalmente para actuar como un transportador de conocimientos involucrado, memorizable y eficiente. La historia es el conocimiento, de forma reelaborada" (Ogborn et al., 2002, p. 102).

Aunque para algunos estudiantes sea difícil asimilar el progreso científico como un camino largo y no lineal, puede ser reconfortante para ellos descubrir que cada nuevo descubrimiento o concepto en biología no se produce en solitario. Por lo tanto, al compartir y analizar la historia de la biología con los educandos, el docente presenta una visión más realista de la construcción del conocimiento biológico, con todas las hipótesis en conflicto, los resultados contradictorios, las limitaciones tecnológicas, los errores y retrocesos, y también, con las influencias externas, tales como la cultura y la política que afectan conjuntamente la dirección de cualquier investigación. En otras palabras, "la historia de la ciencia nos ha enseñando que el estado de ella, en un momento dado es el resultado de una convergencia de múltiples caminos, enfoques $u$ condiciones... No hay una ruta continua y recta, sino ires y venires, desviaciones y reencuentros, cambios en el nivel de análisis, reformulaciones y readecuaciones metodológicas" (Valbuena et al., 2007, p. 147). Lo anterior promueve en los estudiantes el desarrollo del pensamiento crítico, a través de ambientes de aprendizaje en los cuales ellos se sienten cómodos exponiendo sus propias concepciones erradas, arriesgándose y preguntando.

La integración de la realidad social y de la historia de la biología con los contenidos disciplinares de esta ciencia, además de enriquecer los contenidos y desarrollar una actitud crítica, fomenta el pensamiento de los estudiantes a través de la comparación y la metáfora proporcionando "... un marco dentro del cual pueden formularse preguntas productivas y pueden 
extraerse respuestas sin necesidad de conocerlo todo... El alumno puede observar mejor lo que necesita conocer o comprender" (Ogborn et al., 2002, p. 110). De hecho, esta estrategia se ha aplicado exitosamente durante el desarrollo de la disciplina de la biología. "La comparación de Darwin entre la evolución y la cría de animales domésticos implica inmediatamente la pregunta siguiente, "¿quién hace el papel en la evolución de la persona que selecciona los ejemplares en la cría?». De este modo Darwin dirigió sus pasos a la selección natural. La comparación y la metáfora funcionan de este modo gracias a su poder de evocación y de imaginación. Ese poder deriva de su naturaleza concreta, que permite al pensamiento ponerse a trabajar sin conocer aún todo lo que necesita saber" (Ogborn et al., 2002, p. 110).

En este proceso hay que hacer notar que, debido a que el aprendizaje está continuamente en desarrollo, la implementación de un contenido interdisciplinar, basado en los problemas complejos del mundo real, debe ser considerada cuidadosamente en todas sus implicaciones y consecuencias, buscando la mejor manera de poder observar cómo estos contenidos influyen en el desempeño de los estudiantes. Es decir, hay que superar el problema de que “...la evaluación no informe realmente sobre lo que el alumno verdaderamente sabe, sino sobre lo que aparenta que sabe" (Porlán, 1998, p. 181).

Por ello, los educadores deben tener mucha claridad sobre los resultados específicos que pretenden alcanzar. Sin importar los objetivos particulares que tenga cada enseñante es fundamental que en todos ellos se refleje una postura “... filosófico-ideológica, según la cual la escuela, como institución socializadora, debe formar ciudadanos comprometidos en el esclarecimiento y la gestión de los problemas más relevantes del mundo en que viven, con el fin de acceder a una vida más digna, satisfactoria y justa, y ello pasa por la construcción de una visión compleja de la realidad" (García, 1998, p. 84).

Por último, se espera que la integración del contexto social y de la historia de la biología hagan posible un aprendizaje balanceado de los aspectos biológicos básicos, que vaya más allá del simple entretenimiento y que desarrolle en los alumnos la capacidad para seguir estableciendo conexiones entre los diferentes tipos de conocimiento, siendo esta una habilidad esencial para todos los miembros de la sociedad, sean estos biólogos, políticos o cualquier ciudadano que pueda influir en la comunidad local, nacional e internacional. 


\section{BIBLIOGRAFÍA}

- Aikenhead, G.S. (1985). Collective decision making in the social context of science. Science Education, 69 (4), 453-475.

- Arnay, J. (1997). Reflexiones para un debate sobre la construcción del conocimiento en la escuela: Hacia una cultura científica escolar. En: Rodrigo, M.J. y Arnay, J. (compiladores). La construcción del conocimiento escolar. Barcelona: Piados. pp. 35-58.

- Ausubel, D., Novak, J., Hanesian, H. (1978). Educational Psychology: A Cognitive View, $2^{\underline{a}}$ Edición, New York.

- Bingle, W.; Gaskell, P.J. (1994). Scientific Literacy for decision making and the social construction of scientific knowledge. Science Education, 78(2), 185-201.

- Chevallard, Y (1991). ¿Por qué la transposición didáctica? En: La transposición didáctica: Del saber sabio al saber enseñado. Traducción de Claudia Gilman. Argentina: Aique. pp. 11-44.

- García, J.F. (1998). Hacia una teoría alternativa sobre los contenidos escolares. Colección Investigación y Enseñanza. Serie: Fundamentos. Sevilla: Díada Editora S.L.

- Gil, D., Carrascosa, J., Furió, C., Martinez y Torregrosa, J. (1991). La enseñanza de las ciencias en la educación secundaria. Barcelona: Horsori.

- Gil-Pérez, D., Carrascosa, J., Martínez, F. (2000). La Didáctica de las Ciencias. Una disciplina emergente y un campo específico de investigación. En: Perales, F., Cañal, P. Didáctica de las Ciencias Experimentales. Teoría y práctica de la Enseñanza de las Ciencias. Alcoy: Marfil, p.p. 11-34.

- Lattuca, L., Voigt, L., Fath, Q. (2004). Does interdisciplinarity promote learning? Theoretical support and researchable questions. Rev. Higher Educ. 28, 23-48.

- NRC (2005). Facilitating Interdisciplinary Research, Washington, D.C.: National Academy of Sciences.

- Ogborn, J., Kress, G., Martins, I., Mcgillicuddy, K. (2002). La reelaboración de los conocimientos. En: Formas de explicar la enseñanza de las ciencias en Secundaria. Madrid: Santillana. pp. 89-114.

- Porlán, R. (1998) Pasado, presente y futuro de las ciencias. Enseñanza de las Ciencias 16 (1), 175-185.

- Solbes, J., Vilches, A. (1997) STS interactions and the teaching of Physics and Chemistry. Science Education, 81(4), 337-386.

- Valbuena, E., Sierra, C.A., Gutierrez, A.M. (2007). Una década de investigación en la Facultad de Ciencia y Tecnología de la Universidad Pedagógica Nacional. 1. Desarrollo de los procesos investigativos en el Departamento de Biología de la Universidad Pedagógica Nacional. Aportes a la producción de conocimiento, la formación docente y la proyección social. TEA 22, 146-189. 
- Vygotsky, L. (1978). Mind in Society: The Development of Higher Psychological Processes. Cambridge: Harvard University Press. 